\title{
OS DISTÚRBIOS FONOARTICULATÓRIOS NA SÍNDROME DE DOWN E A INTERVENÇÃO PRECOCE
}

\section{The inarticulateness distress in Down syndrome and early intervention}

\author{
Lívia Fernandes Barata ${ }^{(1)}$, Anete Branco ${ }^{(2)}$
}

\begin{abstract}
RESUMO
Tema: a criança com Síndrome de Down apresenta um atraso na aquisição e desenvolvimento dos aspectos fonoarticulatórios, atraso este, que tem sido atribuído a características físicas e ambientais que influenciam negativamente no processo de desenvolvimento. Caracterizar os aspectos fonoarticulatórios dos sujeitos com Síndrome de Down pode proporcionar uma melhor compreensão das alterações abordadas na reabilitação em suas diversas modalidades e contribuir na evolução terapêutica. Por esta razão decidiu-se focar com maior profundidade em um dos aspectos mais importantes da comunicação, a fonoarticulação. Objetivo: caracterizar as alterações fonoarticulatórias encontradas em indivíduos portadores da Síndrome de Down, por meio de uma revisão bibliográfica, enfocando a importância da intervenção precoce frente a tais circunstâncias. Conclusão: a falta de estimulação e o encaminhamento tardio irão interferir no desenvolvimento fonoarticulatório, assim, por meio da intervenção precoce, será possível a obtenção de melhores resultados, fazendo com que grandes problemas tornem-se alterações mínimas.
\end{abstract}

DESCRITORES: Síndrome de Down; Distúrbios da Fala; Diagnóstico

\section{INTRODUÇÃO}

A John Down foi atribuída a primeira descrição pormenorizada e criteriosa de um distúrbio denominado mongolismo ou idiotia mongoloide, supondo que muitos dos sintomas presentes no paciente que estava estudando, fossem típicos do grupo étnico mongol ${ }^{1}$. Com o passar do tempo, tal ponto de vista foi descartado em função do rótulo racialmente pejorativo de mongolismo, dando lugar à designação atual de Síndrome de Down (SD) ${ }^{2}$.

Descobriu-se ao longo dos anos que esses indivíduos possuíam um cromossomo extra, ao invés de dois cromossomos 21, o que levou ao termo Trissomia do $21^{3}$.

(1) Fonoaudióloga Clínica; Instituto de Gerenciamento em Deglutição, IGD, São Paulo, SP, Brasil; Especialização em Motricidade Orofacial em Oncologia pelo Hospital do Câncer AC Camargo; Especialização em Voz pelo Centro de Estudos da Voz; Mestranda na área de Ciências em Oncologia no Hospital AC Camargo.

(2) Fonoaudióloga Clínica; Docente do CEFAC - Pós-Graduação em Saúde e Educação; Mestre em Pediatria pela Faculdade de Medicina de Botucatu da Universidade Estadual Paulista; Doutoranda na área de Ciências em Oncologia no Hospital AC Camargo.

Conflito de interesses: inexistente
No Brasil, estima-se que ocorra um caso em cada 600 nascimentos, o que significa que nascem 8 mil bebês com SD por ano. A estimativa é de que vivem no país 300 mil pessoas que nasceram com a SD ${ }^{4}$.

Até mesmo para leigos no assunto, a face do portador da SD é facilmente identificada, sendo que, na literatura, ela é a mais típica das síndromes descritas. O rosto da criança portadora da SD apresenta um contorno achatado devido, principalmente, aos ossos faciais pouco desenvolvidos. As pálpebras são estreitas e levemente oblíquas; as orelhas são pequenas. O pescoço pode ter uma aparência larga e grossa ${ }^{2}$.

O paciente apresenta cavidade oral de tamanho reduzido, alterações nos órgãos que compõem o sistema estomatognático, ocasionando distúrbios fonoarticulatórios. A hipotonia muscular provoca um desequilíbrio de forças entre os músculos orais e faciais, alterando a arcada dentária, dando um aspecto de projeção mandibular e contribuindo para que a língua assuma uma posição inadequada. A respiração oral, além de deixar a criança mais suscetível a infecções respiratórias, altera seu palato e dificulta a articulação dos sons, sendo a fala um dos maiores problemas existentes nestes indivíduos. 
As principais características que os predispõem às dificuldades com a fala são: hipotonia e a respiração oral ${ }^{5}$.

Pela diversidade da problemática encontrada nos pacientes com SD, é necessário que os pais e os profissionais da saúde atuem com muita atenção no sistema estomatognático (SE), neste caso, no sentido de estimular as funções fisiológicas, sendo que, com o crescimento crânio-facial encerrado e a musculatura aderida às bases ósseas, os resultados poderão ser parciais. Faz-se necessário o acompanhamento continuado, dedicado e precoce ao bebê, pois o indivíduo portador da SD pode apresentar alterações significativas durante seu crescimento e desenvolvimento, tanto em nível orgânico, fisiológico, como cognitivo ${ }^{6}$.

Esta pesquisa tem o intuito de descrever, por meio de uma revisão bibliográfica, os distúrbios fonoarticulatórios causados pela trissomia do cromossomo 21 e evidenciar a importância da intervenção fonoaudiológica precoce sobre eles, a fim de minimizar os efeitos e limitações das alterações no sistema de fonoarticulação do paciente com SD, possibilitando uma integração e aceitação efetiva do indivíduo na sociedade. Embora o enfoque terapêutico não seja o objetivo deste estudo, é importante mencionar que uma abordagem multidisciplinar é fundamental para o mais apropriado tratamento destes pacientes.

Caracterizar os aspectos fonoarticulatórios dos sujeitos com Síndrome de Down pode proporcionar uma melhor compreensão das alterações abordadas na reabilitação em suas diversas modalidades e contribuir em sua evolução terapêutica. No entanto, existem diferenças marcantes tanto no que diz respeito às alterações fonoarticulatórias quanto em outros aspectos da comunicação, tais como aquisição da linguagem e déficit auditivo. Estudálos em conjunto não permitiria uma compreensão em profundidade de particularidades que podem ser importantes em tal caracterização. Por esta razão decidiu-se focar em um dos aspectos mais importantes da comunicação, a fonoarticulação.

\section{MÉTODOS}

A fim de atender aos objetivos propostos, elaborou-se um estudo exploratório descritivo, feito através de um levantamento bibliográfico, dos últimos 50 anos (1959-2009), utilizando-se as bases de dados LILACS e PUBMED, livros (encontrados em bibliotecas de instituições de ensino e pesquisa) e periódicos da área, nos idiomas português, inglês e espanhol. Foram estabelecidos para a busca os termos Síndrome de Down; linguagem; motricidade oral; distúrbios de voz e fala; diagnóstico e intervenção precoce.

Os critérios para inclusão das referências bibliográficas na pesquisa foram distintos. Os textos deveriam conter as palavras-chave supra citadas; relacionando o sujeito com Síndrome de Down em tendências e pesquisas.

Realizou-se um corte histórico para delimitar o número de dados do estudo, incluindo-se publicações que iam desde o início dos anos 50 até os dias atuais, pela necessidade de se conhecerem e se confrontarem as tendências das referidas épocas.

Após identificação, os documentos foram agrupados por ordem cronológica, temática discutida e enfoque, facilitando, assim, a análise, o que permitiria conhecer as perspectivas das pesquisas na Síndrome de Down.

Ao final do levantamento, obteve-se um total global de 24 textos.

Depois de realizadas as etapas descritas, iniciou-se a redação do presente trabalho de revisão, tornando possível articular as diferentes representações que propiciaram o entendimento sobre 0 assunto.

\section{REVISÃO DA LITERATURA}

As alterações fonoarticulatórias referem-se a problemas de execução motora que podem comprometer além da produção fonatória, a respiração, a ressonância, a articulação e a prosódia. Os fatores que intervêm no processo articulatório são ${ }^{7}$ :

1) Função auditiva: responsável pelo fornecimento do modelo acústico (recepção, discriminação e retenção), vindo do exterior e do próprio indivíduo (feedback auditivo).

2) Função tátil: responsável pela informação sobre os pontos de contato durante a articulação (feedback táti).

3) Função proprioceptiva: informa sobre as sensações dos músculos e tendões, fornecendo condições para análise do movimento articulatório, da pressão no momento do contato e da tonicidade da musculatura envolvida (feedback cinestésico).

4) Função visual: responsável pela formação de modelos visuais das produções articulatórias.

Qualquer desvio que atinja um ou mais órgãos utilizados no aspecto fonoarticulatório causará dificuldades ou impedimentos na articulação e alterações sobre a fonação. $O$ desenvolvimento das habilidades fonoarticulatórias pode ser precursor necessário para as habilidades de linguagem, ou seja, desordens ao nível de linguagem e disfunção do sistema sensório motor oral, frequentemente ocorrem juntos ${ }^{8}$. 
A maioria dos portadores da SD apresenta face curta, sendo poucos os casos de mesio e dolicofaciais ${ }^{5,9}$. Os achados cefalométricos demonstram a existência de um déficit significativo de crescimento da maxila, estando de acordo com a alta incidência de classe III, mordidas cruzadas uni e bilaterais e mordida aberta ${ }^{5,9,10}$.

O tamanho médio da mandíbula na SD não é, significativamente, maior do que nas pessoas normais. O que ocorre é um posicionamento anterior da face. O crescimento e o desenvolvimento anormais da mandíbula podem também ser causados pela hipotonia dos músculos temporal e masseter, hiperfuncionalidade das articulações temporomandibulares, respiração oral, língua alojada na parte anterior da cavidade oral, modo adaptado de deglutir, além do insuficiente crescimento ântero-posterior e vertical. A classe III também pode ser resultado da função e postura da língua. Alguns casos de classe III são verdadeiros casos de prognatismo ${ }^{9}$. Muitos fatores estão associados à mordida aberta anterior, como crescimento deficiente da maxila acompanhado do pressionamento da língua e doenças periodontais ${ }^{11}$.

Por outro lado, em um estudo brasileiro realizado com 102 crianças com SD, de ambos os sexos, e com faixa etária entre 3 a 16 anos, a maioria dos pacientes apresentou desenvolvimento dentário dentro do padrão de normalidade ${ }^{12}$.

Quanto à hipotonia, quando acentuada, pode ocasionar uma menor movimentação dos órgãos fonoarticulatórios (OFAs), refletindo em imprecisões articulatórias, substituições ou distorções de sons. Além da hipotonia e hipomobilidade, a falha na propriocepção de lábios pode levar à omissão ou distorção dos sons bilabiais. A protrusão da língua, encontrada na SD, pode causar ceceio: a hipotonia de dorso de língua tem como consequência alterações ou omissão dos fonemas linguopalatais e os problemas de mobilidade de ponta de língua podem ocasionar a má articulação ou omissão do /r/ brando e de outros sons linguodentais e linguoalveolares ${ }^{13}$.

Outra alteração encontrada nestes indivíduos é a mordida aberta e, quanto a isso, a articulação dos fonemas linguodentais, nesses casos, são articulados como interdentais. O ceceio anterior nesse contexto fica facilitado, contribuindo para a distorção desses fonemas. Na classe III, observa que a articulação é imprecisa, os fonemas linguodentais $/ \mathrm{n}, \mathrm{l}, \mathrm{t}, \mathrm{d} / \mathrm{são}$ produzidos com a ponta da língua atrás dos dentes inferiores e a parte medial fazendo contato com a papila retro-incisal. Os fonemas fricativos /f, v/ são pronunciados com o lábio superior e incisivos inferiores, indicando que as alterações de ponto de articulação acompanham as alterações anatômicas ${ }^{13}$.
O choro de uma criança com Síndrome de Down é monótono, grave e áspero. A voz de uma criança maior com esta síndrome pode ser tensa, rouca ou gutural ${ }^{14}$. Indivíduos portadores desta síndrome, além de apresentarem soprosidade e rouquidão, têm também uma forte prevalência de hipernasalidade. As alterações de ressonância encontradas nestes pacientes devem-se, principalmente, à forma alterada das cavidades de ressonância, agravada pela imprecisão do movimento de órgãos fonoarticulatórios ${ }^{15}$.

Nos achados da literatura, foram encontrados espessamento de mucosa das pregas vocais. A mucosa faríngea apresentou sinais de atrofia e tendência ao ressecamento, hipertrofia das amígdalas e palato profundo. A movimentação do palato mole e das pregas vocais é normal. A qualidade vocal tensa ou estridente foi atribuída à hipertonicidade das pregas vestibulares durante a fonação ${ }^{15}$.

A qualidade vocal dos indivíduos com SD apresentam frequência fundamental mais baixa (devido a provável falta de controle em tensionar as pregas vocais, alterando a frequência de vibração), acompanhada por instabilidade. Jitter e shimmer são correlacionados com os desvios encontrados, incluindo rugosidade, soprosidade, astenia e aspereza ${ }^{16}$. É importante ressaltar que uma voz instável pode comprometer a inteligibilidade da mensagem, além disso, tal parâmetro auditivo apresenta uma conotação psicodinâmica negativa, tornando o falante subestimado em suas reais capacidades ${ }^{14,17}$.

Outro fator orgânico que influencia na comunicação são os déficits sensoriais, como a deficiência auditiva. Uma causa relevante de comprometimento auditivo nesta população é representada pela otite média e anomalias otológicas em associação com a síndrome, como as malformações dos ossículos da orelha média e as aspirais cocleares encurtadas. Diferenças nas estruturas neurais do tronco cerebral também podem afetar a transmissão do estímulo auditivo ao longo do trajeto do VIII par do nervo craniano. Tal deficiência prejudica, significativamente, a automonitoração do portador da síndrome em níveis fonoarticulatórios e prosódicos. É por meio do feedback auditivo que a criança regula a qualidade da articulação, compreende o som, pode corrigir-se na fala, na escrita e na leitura ${ }^{5}$.

É importante sanar ou minimizar aspectos físicos que possam prejudicar a articulação e produção vocal por meio de exercícios para fortalecimento da musculatura, adaptação de próteses dentárias ou auditivas ${ }^{2}$.

A linguagem representa um dos aspectos mais importantes a ser desenvolvido por qualquer criança, para que possa se relacionar com as demais pessoas e se integrar no meio social. Indivíduos mais 
hábeis na linguagem podem comunicar melhor seus sentimentos, desejos e pensamentos. Desenvolver somente habilidades motoras orais pode não ser suficiente para que as crianças adquiram fala e linguagem; elas precisam compreender que os sons são instrumentos utilizados na comunicação e estes serão os grandes elementos de motivação para que elas empreguem seus esforços com o intuito de aprender a articular os sons da fala, a fim de permitir, ou garantir, a interação criança-adulto, caracterizando sua função comunicativa e social. Estas trocas comunicativas propiciam a compreensão da linguagem pela criança, a atribuição de significado às suas emissões, a aprendizagem e o desenvolvimento cognitivo ${ }^{2}$. A participação dos pais é fundamental para maximizar o desenvolvimento motor por meio da rotina diária da criança ${ }^{18}$.

Entre as limitações cognitivas usualmente citadas e as deficiências dos pacientes com a SD, acham-se a duração curta da atenção, o tempo lento de reação, a deficiência no processamento auditivo-vocal, a limitação da memória de fatos recentes (variando de acordo com aspectos distintos da informação sensorial), diminuição da discriminação perceptiva e da capacidade de generalização e a deficiência da facilidade de simbolização ${ }^{5}$.

Ser estimulada precocemente é fundamental para o desempenho futuro da criança, tenha ela ou não SD. Toda a sistematização do conhecimento humano ocorre através do fenômeno da mielinização. A mielina funciona como um condutor elétrico da informação neural e só se forma a partir da soma de dois fatores: um interno e um externo. $O$ fator interno depende de uma constituição orgânica saudável e eficiente. $\mathrm{O}$ externo, de estímulos percebidos através dos cinco sentidos e experiências motoras. Dele fazem parte os fatores ambientais, nos quais se inclui uma alimentação correta, estímulos táteis e visuais variados, afeto, entre outros. Portanto, se a criança que nasce com a síndrome tem uma estrutura interna deficitária, a estimulação adquire uma importância ainda maior ${ }^{2}$.

É imprescindível a estimulação antes dos dois anos de idade, pois o sistema nervoso central está em formação, nesse período. O trabalho será ainda mais satisfatório quando iniciado mais próximo ao nascimento, se possível com orientações no próprio hospital, diminuindo, assim, as chances de que comportamentos adaptativos se instalem. Neste período, as orientações em relação à alimentação em conjunto com o trabalho miofuncional poderão proporcionar um desenvolvimento favorável das estruturas da face ${ }^{2}$.

A deficiência mental está relacionada a uma lentidão um pouco maior de aprendizado e obtenção de conhecimento. A dificuldade se dá em desenvolver o lado simbólico, ou seja, conceitos abstratos como passado e futuro. Assim, o aprendizado é muitas vezes realizado por meio dos sentidos. Por isso, todo o tipo de estimulação é a chave para a vida mais saudável e rica em experiências ${ }^{4}$. Além disso, existe uma evidência de que crianças com SD mostram diferenças no processamento da informação em seu desenvolvimento ${ }^{19-21}$.

A estimulação precoce objetiva o desenvolvimento de estruturas cerebrais, que responderão pelas atividades psicomotoras cada vez mais complexas de forma gradativa. As diferentes áreas que devem ser estimuladas dizem respeito às funções motoras, sensoriais, à fala e aspectos cognitivos ${ }^{21}$ :

\section{1) Estimulação das funções motoras}

- Propriocepção: sensação de onde se localizam partes do seu próprio corpo, no espaço, com maior diversidade de experiências sensitivas e sensoriais através do rolar, movimentar braços e pernas, praxia do sistema sensório motor oral e do próprio toque.

- Motricidade: movimentos diversos estimulados e reforçados, que propiciam tonicidade e força muscular adequadas. Este é um trabalho que resulta na consciência do próprio corpo e inibição de movimentos estereotipados.

\section{2) Estimulação Sensorial}

- Visual: seleção visual e atenção, estimulando a exploração e manipulação dos objetos que as rodeia.

- Auditiva: localização sonora a fim de exercitar a memória, atenção e a repetição de sons. Imitando os sons, possibilita-se a repetição por parte da criança e início de um jogo que será importante para a futura articulação da fala. A audição começa com a discriminação dos sons. Os sons que a criança é capaz de emitir são muito ricos e variados e, por essa razão, devese dar oportunidade de exercitar os movimentos de boca e lábios constantemente.

\section{3) Estimulação da fala}

A melhora da sensibilidade e o treinamento dos movimentos dos órgãos fonoarticulatórios fazemse extremamente necessários para a consequente adequação de tônus, postura e mobilidade, que desempenhará função preparatória para a coordenação envolvida na futura articulação da fala. Assim sendo, as manobras que visam à aceitação de estímulos, a conscientização da região trabalhada e o reconhecimento oral, favorecem o equilíbrio do tônus e da postura e, consequentemente, a realização dos movimentos específicos dos OFAS. Como algumas crianças têm tendência a permanecer com 
a língua anteriorizada, pode-se trabalhar tônus e postura dos lábios, propiciando o vedamento labial e adequação de suas funções; correções da hipotonia de bochechas, responsável para a manutenção de uma pressão intra-oral diminuída e uma menor energia na produção dos fonemas oclusivos e fricativos; mobilidade do palato mole que se encontra incompetente na SD; exercícios que facilitem a postura e tensão da língua, evitando a protrusão, devido à hipotensão ${ }^{21}$.

Da mesma maneira que todos os outros movimentos da criança com SD são incoordenados e demoram a evoluir satisfatoriamente, a hipofuncionalidade da língua dificulta sua posição intra-oral, necessária para emissão precisa dos linguodentais $(/ / /, / \mathrm{n} /, / \mathrm{d} /, / \mathrm{r} /, / \mathrm{t} /)$. Os bilabiais são mais fáceis de serem reproduzidos e observados (geralmente os primeiros que surgem ao longo do desenvolvimento). Esta é a razão da importância do auxílio do fonoaudiólogo, pois as orientações necessárias serão dadas através deste profissional, para que os adultos não façam exigências, solicitando à criança sons muito complexos, que ainda não poderão ser emitidos ${ }^{13,21}$.

$\mathrm{Na}$ SD, desde o nascimento, são encontrados distúrbios relacionados com a comunicação, principalmente no que se refere à expressão. A criança encontra dificuldade para sugar, deglutir ${ }^{22}$, mastigar, controlar os movimentos dos lábios e da língua e isto ocasiona atraso na articulação dos movimentos que compõem a fala expressiva. $O$ trabalho terapêutico visa nos primeiros anos de vida, melhorar as funções de sucção, mastigação, deglutição, respiração e fonação, que atuam como pré-requisitos para a aquisição da fala. Esse trabalho de base torna-se importante devido à hipotonia ${ }^{21}$.

Além de atuar na construção da linguagem (nãoverbal e verbal) a Fonoaudiologia segue as etapas de desenvolvimento da criança e de sua interação com o meio, visando a aquisição de uma fala clara e bem-articulada, dentro de certos limites, a qual incentiva a interação e a comunicação social, contribuindo para o desenvolvimento da articulação dos sons e da habilidade verbal, bem como orientando a família para as maneiras adequadas de estimulação ${ }^{21,23,24}$.

O trabalho dos profissionais é bem mais abrangente do que simplesmente orientar os pais sobre como devem segurar ou brincar com o bebê. A estimulação engloba uma série de práticas específicas usadas para desenvolver a capacidade da criança de acordo com seu grau de comprometimento e da fase de desenvolvimento em que se encontra ${ }^{21}$.

Os benefícios potenciais obtidos para a área, com esta pesquisa de revisão de literatura, incluem a possibilidade de aquisição de informações que poderão resultar em maior conhecimento sobre as alterações encontradas nos pacientes com Síndrome de Down, valorizando assim o indivíduo no seu processo de reabilitação. Assim, conhecendo tais distúrbios podem minimizar o real impacto da alteração da comunicação em sua qualidade de vida.

\section{CONCLUSÃO}

A falta de estimulação e o encaminhamento tardio irão interferir no desenvolvimento fonoarticulatório, assim, por meio da intervenção precoce, será possível a obtenção de melhores resultados, fazendo com que grandes problemas tornem-se alterações mínimas ${ }^{19}$.

Caracterizar os aspectos fonoarticulatórios dos sujeitos com Síndrome de Down pode proporcionar uma melhor compreensão das alterações inerente a essa população, podendo estas ser abordadas na reabilitação em suas diversas modalidades, servindo como parâmetro na evolução terapêutica.

\section{ABSTRACT}

Background: children with Down's Syndrome show a retard in language acquisition and develop inarticulateness distress. This delay has been attributed to the physical and environmental characteristics that have a negative impact on the development process. Characterizing articulation aspects of subjects with Down Syndrome may provide a better understanding of the changes addressed in rehabilitation in their different ways and contribute to the development therapy. For this reason, we decided to focus in greater depth in one of the most important communication aspects, the articulation. Purpose: characterize the inarticulateness distress found in subjects with Down Syndrome by means of a literature review, focusing on the importance of early intervention before such circumstances. Conclusion: lack of stimulation or late treatment will affect the development phonoarticulatory, thus, through early intervention it will be possible to obtain better results, causing major problems to become minimal disturbances.

KEYWORDS: Down Syndrome; Speech Disorders; Diagnosis 


\section{REFERÊNCIAS}

1. Down JLH. Observations on an ethnic classification of idiots. London Hospital Reports. 1866; 3:259-62. 2. Werneck C. Muito prazer, eu existo. Rio de Janeiro: WVA; 1993.

3. Lejeune J, Turpin R, Gautier MM. Le mongolisme premier exemple d'aberration autosomique humaine. Annee Génétique. 1959; 1(2):41-9.

4. Frasão Y. Down: uma nova e surpreendente visão. Rev Fonoaudiol. 2007; (72):10-4.

5. Bishop D, Mogford K. Desenvolvimento da linguagem em circunstâncias excepcionais. Rio de Janeiro: Revinter; 2002. p. 1-26.

6. Carvalho GD. S.O.S. Respirador bucal. São Paulo: Lovise; 2003.

7. Cohen MM, Winer RA. Dental and facial characteristics in Down's syndrome (Mongolism). J Dent Res. 1965; 44(Suppl):197-208.

8. Fischer-Brandies H. Cephalometric comparison between children with and without Down's syndrome. Eur J Orthod. 1988; 10(3):255-63.

9. Jensen GM, Cleall JF, Yip ASG. Dentoalveolar morphology and developmental changes in Down's syndrome (trisomy 21). Am J Orthod. 1973; 64(6):607-18.

10. Moraes MEL, Bastos MS, Santos LRA, Castilho JCM, Moraes LC, Médici Filho E. Dental age in patients with Down syndrome. Braz Oral Res. 2007; 21(3):259-64.

11. Spinelli VP, Massari IC, Trenche MCB. Distúrbios articulatórios. In: Ferreira LP, Barros MCPP, Gomes ICD, Proença MG, Limongi SCO, Spinelli VP, et al. Temas de fonoaudiologia. São Paulo: Loyola; 2002. p.123-97.

12. Alcock K. The development of oral motor control and language. Down Syndr Res Pact. 2006; 11(1):1-8.

13. Limongi SCO, Gomes ICD, Proença MG. Avaliação e terapia da motricidade oral. In:
Ferreira LP, Barros MCPP, Gomes ICD, Proença MG, Limongi SCO, Spinelli VP, et al. Temas de fonoaudiologia. São Paulo: Loyola; 2002. p. 61-119. 14. Behlau M, Thomé R, Azevedo R, Rehder MI, Thomé DC. Disfonias congênitas. In: Behlau M. Voz: o livro do especialista. Vol. 2. São Paulo: Revinter; 2005. p.1-34.

15. Montague J, Hollien $\mathrm{H}$. Perceived voice quality disorders in Down's syndrome children. J Commun Disord. 1973; 6:76-87.

16. Moura CP, Cunha LM, Vilarinho $\mathrm{H}$, Cunha MJ, Freitas D, Palha M, et al. Voice parameters in children with Down syndrome. J Voice. 2008; 22(1):34-42.

17. Lee MT, Thorpe J, Verhoeven J. Intonation and phonation in young adults with Down syndrome. $\mathrm{J}$ Voice. 2009; 23(1):82-7.

18. Gisel EG. Effect of food texture on the development of chewing of children between six month and two years of age. Dev Med Child Neurol. 1991; 33(1):69-79.

19. Virji-Babul N, Kerns K, Zhou E, Kapur A, Shiffrar M. Perceptual-motor deficits in children with Down syndrome: implications for intervention. Down Syndr Res Pract. 2006; 10(2):74-82.

20. Mahoney G, Perales F. The role of parents in early motor intervention. Down Syndr Res Pract. 2006; 10(2):67-73.

21. Lefèvre $\mathrm{BH}$. Mongolismo: orientação para famílias. Compreender e estimular a criança deficiente. São Paulo: Almed; 1988.

22. Bastos DC. A conduta vocal dos portadores de Síndrome de Down. Rev Fono Atual. 2001; (16):39-44.

23. Dada S, Alant E. The effect of aided language stimulation on vocabulary acquisition in children with little or no functional speech. Am J Speech Lang Pathol. 2009; 18(1):50-64.

24. Porto-Cunha E, Limongi SCO. Communicative profile used by children with Down syndrome. PróFono. 2008; 20(4):243-8.

RECEBIDO EM: 31/03/2008

ACEITO EM: 11/11/2009

Endereço para correspondência:

Lívia Barata

Rua Tamandaré, 246/203

São Paulo - SP

CEP: 01525-000

E-mail: liviabarata@gmail.com 